This item was submitted to Loughborough's Research Repository by the author.

Items in Figshare are protected by copyright, with all rights reserved, unless otherwise indicated.

\title{
An investigation of the growth of bismuth whiskers and nanowires during physical vapour deposition
}

PLEASE CITE THE PUBLISHED VERSION

http://dx.doi.org/10.1088/0022-3727/45/43/435304

PUBLISHER

(c) IOP Publishing Ltd

VERSION

AM (Accepted Manuscript)

LICENCE

CC BY-NC-ND 4.0

\section{REPOSITORY RECORD}

Stanley, Steven A., Christopher Stuttle, Andrew J. Caruana, Michael D. Cropper, and A.S.O. Walton. 2019. "An Investigation of the Growth of Bismuth Whiskers and Nanowires During Physical Vapour Deposition". figshare. https://hdl.handle.net/2134/10739. 
This item was submitted to Loughborough's Institutional Repository (https://dspace.lboro.ac.uk/) by the author and is made available under the following Creative Commons Licence conditions.

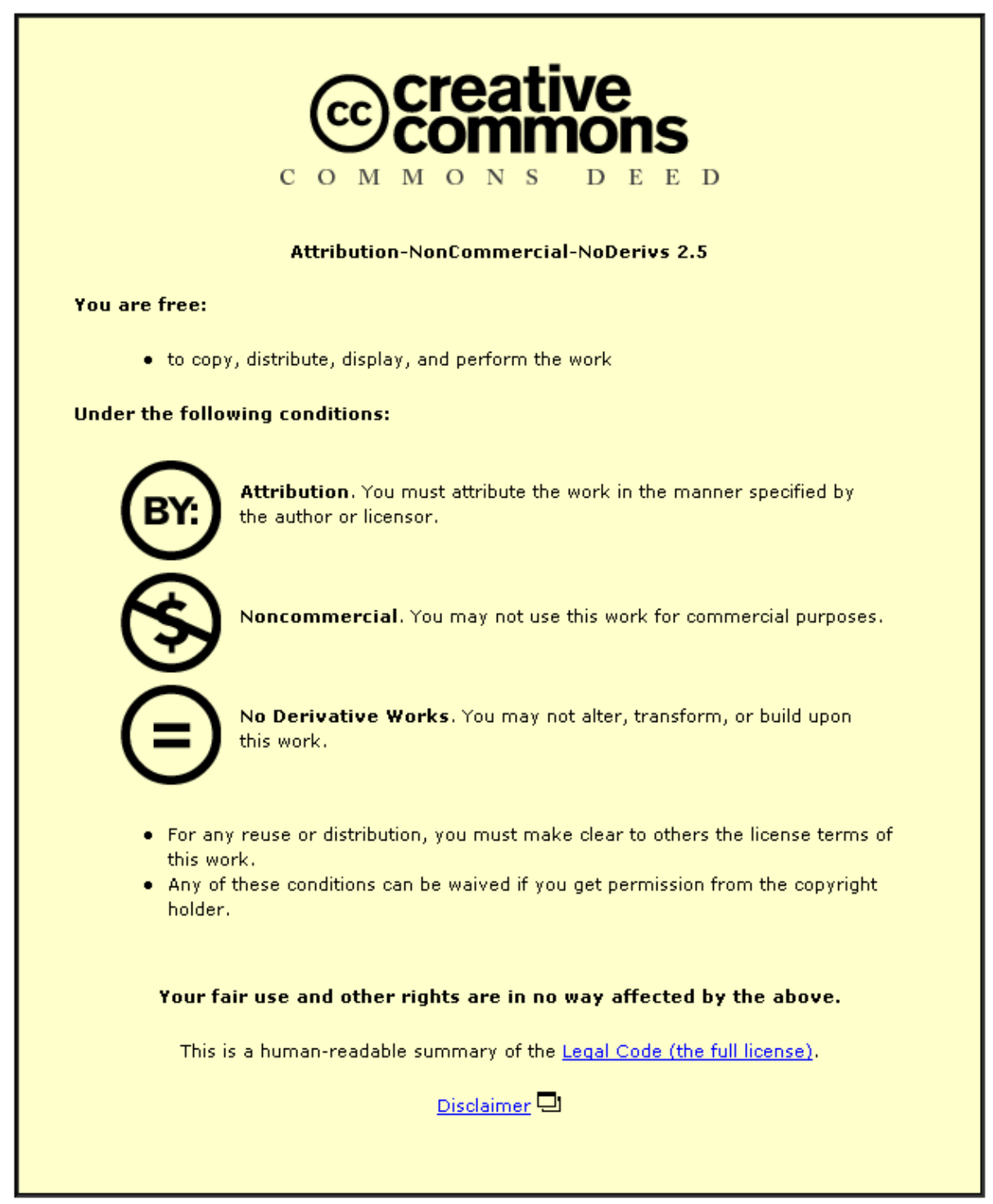

For the full text of this licence, please go to: http://creativecommons.org/licenses/by-nc-nd/2.5/ 


\title{
An investigation of the growth of bismuth whiskers and nanowires during physical vapour deposition
}

\author{
S A Stanley ${ }^{\mathrm{a}}$, C Stuttle ${ }^{\mathrm{a}, \mathrm{b}}$, A J Caruana ${ }^{\mathrm{a}}$, M D Cropper ${ }^{\mathrm{a}}$, A S O Walton ${ }^{\mathrm{c}}$ \\ ${ }^{a}$ Department of Physics, Loughborough University, Loughborough, LE11 3TU, UK \\ ${ }^{\text {b} P r e s e n t ~ A d d r e s s: ~ D e p a r t m e n t ~ o f ~ M a t e r i a l s, ~ L o u g h b o r o u g h ~ U n i v e r s i t y, ~ L o u g h b o r o u g h, ~ L E 11 ~ 3 T U, ~ U K ~}$ \\ ${ }^{\mathrm{c}}$ LENNF, School of Physics and Astronomy, University of Leeds, Woodhouse Lane, Leeds LS2 9JT, UK \\ Email: m.d.cropper@lboro.ac.uk
}

Short Title: Bismuth Whiskers

PACS: 68.70.+w; 81.07.Gf; 61.46.Km; 62.23; 81.15.-z; 81.15.Cd.

\begin{abstract}
Bismuth thin films of thickness in the region of $500 \mathrm{~nm}$ have been prepared by planar magnetron sputtering onto glass, silicon and GaAs substrates. Electron microscopy of these films reveals that bismuth whiskers grow spontaneously when the substrate is heated to temperatures between $110^{\circ} \mathrm{C}$ and $140^{\circ} \mathrm{C}$ during deposition and the optimum temperature for such growth is largely independent of substrate. Depositing films under similar conditions using thermal evaporation does not, however, produce the whisker growth. X-ray diffraction has been employed to investigate film texture with temperature and it has been shown that the film crystallites are predominantly [110] and [111] oriented. The [110] orientation of the crystallites dominates at deposition temperatures above $110^{\circ} \mathrm{C}$ for sputter deposition and the [111] at lower temperatures. The optimum temperature for whisker growth coincides with the temperature for the change between predominant orientations. While sputter deposition appears to favour films with crystallite orientation of [110], thermal evaporation favours [111] and has a higher change-over temperature. The whiskers that grow from the film emerge at off-normal angles between $43.3^{\circ}$ and $69.2^{\circ}$ with a mean of $54 \pm 3^{\circ}$. The projected length of whiskers on a $500 \mathrm{~nm}$ film on a GaAs substrate shows a wide distribution to a maximum of more than $100 \mu \mathrm{m}$. The mean projected length for this sample was $16 \pm 1 \mu \mathrm{m}$ and the diameter is around $0.5 \mu \mathrm{m}$. Measurements of the electrical properties of the whiskers at room temperature reveals ohmic behaviour with an estimated resistivity of $2.2 \pm 0.2 \mu \Omega \mathrm{m}$. Detailed examination of scanning electron micrographs, eliminates all growth mechanisms except tip growth by a non-catalysed vapour-solid/vapour-liquid-solid method. By depositing thinner films it is shown that this spontaneous growth of whiskers offers a route to fabricate high quality bismuth nanowires of lengths exceeding $10 \mu \mathrm{m}$.
\end{abstract}




\section{Introduction}

The spontaneous growth of whiskers on the surface of soft metal coatings of materials such as tin and cadmium is long known [1]. These whiskers are single crystal metallic projections that may be several tens of micrometres long and are typically $500 \mathrm{~nm}$ to a few micrometres diameter. The mechanism for the growth of these whiskers has been the subject of extensive debate, with processes such as dislocations causing tip growth [2] and stress relief promoting root growth by dislocations $[3,4]$ being proposed. Although the precise mechanism is still unknown, the stress model is preferred. It is widely believed that the whiskers grow from the root not the tip [5], often grow from a minority crystal orientation [6], are often promoted by post deposition heating and grow over relatively long periods of time. Despite the long history of the study of whisker growth, the area is of high contemporary interest due to the implications for device failure, particularly in view of the move to lead-free solders.

Whiskers are often high-quality, high-aspect ratio materials; a property that they share with nanowires. The production of nanowires or nanorods is an area of high importance in the physics of materials. A nanowire is said to be a material with width within the nanometres regime and of any length; but normally with a high aspect ratio. Nanowires have also been referred to as one dimensional (1D) materials as they allow electron freedom only along a single axis while allowing quantum effects to be shown in the other two dimensions

Bismuth is an interesting element possessing unique properties. It is a semi-metal with a very low carrier density, low electron effective mass and large mean free path. It is reported that the small overlap between the valence and conduction bands means that it may be converted from a semi-metal to a semiconductor by reducing the dimensions of the material [7]. There have been many reports on the production and characterisation of bismuth whiskers, nanorods and nanowires; far too numerous to review here. Recent interest has been motivated both by their potential application as thermoelectrics $[8,9]$ and by fundamental interest in the size effect [10]. There are several methods for their fabrication including pressure injection into a porous alumina template [11, 12]. However, a recent report by Cao et al [13] showed that high quality [110] (in the hexagonal system) oriented nanorods could be produced on bismuth thin films deposited on $\mathrm{Si}(111)$ without the use of a template when elevated growth temperatures were used. The mechanism that they ascribed to this production was vapoursolid tip growth promoted by screw dislocations.. 
In this report, we show that thin films of bismuth exhibit the spontaneous growth of whiskers when deposited at elevated temperatures on a variety of substrates. We investigate the effect of growth conditions on whisker length and profusion and report the measurement of electronic properties. The growth conditions may be optimised to maximise or minimise whisker growth and we show that this method offers a route to manufacture bismuth nanowires of high regularity.

\section{Experimental}

The bismuth thin films were grown in a clean high vacuum (base pressure $2 \times 10^{-8} \mathrm{mbar}$ ) system manufactured by PVD Products Inc. This load-locked system is equipped with both an evaporation source and three three-inch planar magnetron sources, in the sputter-up configuration. There are options to drive the magnetrons by either RF or DC power. The substrate stage may be rotated during deposition and may be heated to $800^{\circ} \mathrm{C}$ using an array of quartz heaters, the process pressure is set using a mass flow controller and the growth rate may be quantified using a quartz crystal monitor.

In the work reported here, the source was a three inch bismuth target of purity 99.99\% supplied by Testbourne Ltd. The deposition employed RF sputtering driven by a Dressler Cesar RF power generator running at powers of $22 \mathrm{~W}$ and $45 \mathrm{~W}$ to give deposition rates of $0.04 \mathrm{nms}^{-1}$ and $0.12 \mathrm{nms}^{-1}$ respectively. Argon process gas with purity 99.9999\% was supplied at a controlled rate, balanced by the throttled turbo-pump to give a process pressure of 10 mTorr. Growth temperatures between room temperature and $160^{\circ} \mathrm{C}$ were measured using a thermocouple and stabilised using a Eurotherm 2408 proportional-integral-derivative controller. During the reported depositions, the substrate was rotated within plane at thirty revolutions per minute. However, depositions preformed with no rotation showed no observable difference in film structure.

The substrates were prepared by ultrasonic cleaning in acetone and subsequently ethanol for eight minutes. The substrates were then dried before placing into the vacuum chamber. Once under vacuum and stabilised for one hour at the deposition temperature the substrates were etched with $0.65 \mathrm{~W} / \mathrm{cm}^{2} \mathrm{RF}$ for $30 \mathrm{~s}$ before commencing deposition.

We have deposited thin films of bismuth onto different substrates and investigated the resulting microstructure using several methods, including scanning electron microscopy and $\mathrm{X}$-ray diffraction. These thin films were observed to exhibit whisker growth, and 
subsequently electrical conductivity measurements were made of some whiskers in-situ on the film using a microscopic four-point method. The structure of the underlying thin films was investigated by X-ray diffraction measurements using a Philips PW113 diffractometer. We employed the Bragg-Brentano geometry to make $\theta-2 \theta$ scans in the $2 \theta$ range between $20^{\circ}$ and $75^{\circ}$. In addition to scans from the films, a reference powder sample scan was made from bismuth source material for evaporation.

Two different electron microscopes were employed for scanning electron imaging; a Leo 1530FE high resolution field emission gun scanning electron microscope, which was used to obtain images using a $5.00 \mathrm{keV}$ primary energy and magnifications between ten- and fiftythousand times, and a FEI Nova 600 Nanolab Dual Beam system, to obtain micrographs at different substrate angles to create three-dimensional images. In the latter case a high resolution field emission electron column running at $10.00 \mathrm{keV}$ with six-thousand times magnification was used to collect three images at angles differing by $5^{\circ}$ each and topographical images with perspective were synthesised from these using the MeX package by Alicona [14]. Both facilities are based in the Loughborough Materials Characterisation Centre, at the Department of Materials, Loughborough University.

Electrical measurements of selected whiskers were made using an Omicron Nanoprobe based at the Leeds EPSRC Nanoscience and Nanotechnology Facility. This instrument comprises four individual scanning tunnelling microscopes guided by a high resolution Gemini field emission gun scanning electron microscope, within an ultra-high vacuum system with base pressure $\sim 10^{-10}$ mbar. By these means it is possible to form reversible, non-destructive electrical contacts to individual nanostructures, enabling two and four probe resistivity measurements.

\section{Results and discussion}

\subsection{Temperature Dependence}

In agreement with Cao et al [13] we have found that whiskers or nanorods grow spontaneously during the deposition of bismuth thin films, when the conditions are appropriate. We have found that whiskers grow on thin films of bismuth deposited onto all of the flat substrates that we have investigated at any temperature up to a maximum temperature that was in part influenced by the specific substrate and the precise deposition parameters. Whiskers were observed on films deposited onto substrates at temperatures between room 
temperature and $145^{\circ} \mathrm{C}$, with the optimum temperature lying between $110^{\circ} \mathrm{C}$ and $125^{\circ} \mathrm{C}$. No whiskers have been observed at temperatures higher than $145^{\circ} \mathrm{C}$. Whisker development is sparse at room temperature and $145^{\circ} \mathrm{C}$ but profuse in the optimum temperature range. It is important to note that the single temperature used by Cao et al falls outside of the range within which we observe whisker growth; however, it is also the case that we are using a lower deposition rate. It is also interesting to note that our observation of the morphological changes in the film with deposition temperature are closer to those observed by Kim et al [15] in their study of RF magnetron sputtering. However, Kim did not observe whisker growth though their experiment differed in that they deposited at a higher rate of around $0.3 \mathrm{nms}^{-1}$.

An example of a bismuth whisker is shown in figure 1(a) and (b). This is a scanning electron microscope image of a single whisker on a bismuth film grown by RF sputtering onto glass. The film is $400 \mathrm{~nm}$ thick and was deposited at a rate of $0.04 \mathrm{nms}^{-1}$ with a substrate temperature of $145^{\circ} \mathrm{C}$. Figure 1(a) shows the whisker growing from a hillock on a polycrystalline film. Figure 1(b) shows an enlargement of the base of the whisker. There are other hillocks in the surrounding area. It was found that sometimes the whiskers grew from hillocks but that at other times protruded from the film itself. This whisker illustrates several of the key features shared by the whiskers: it is very straight, it has a constant cross-section, it has a diameter comparable with the typical grain size on the film and it has grown at an angle away from normal. The straightness and regularity is quite remarkable when compared with other whiskers in other materials.

To illustrate how profuse whisker growth is at a more favourable temperature, Figure 1(c) shows a lower magnification image of a $500 \mathrm{~nm}$ film grown at $0.12 \mathrm{nms}^{-1}$ on a glass substrate held at $125^{\circ} \mathrm{C}$ during deposition. It can be seen that there are innumerable whiskers, of various lengths and that have grown at essentially random azimuthal angles but closer inspection shows that they rarely emerge at normal or at near-normal angles. The length of these whiskers often exceeds $10 \mu \mathrm{m}$.

Figure 2 shows some further scanning electron micrographs of $500 \mathrm{~nm}$ thickness bismuth films grown by RF sputtering at $0.04 \mathrm{nms}^{-1}$ onto glass substrates held at different temperatures. Figure 2(a) shows a film deposited with the substrate at $30^{\circ} \mathrm{C}$. No whiskers are apparent on this area of the substrate but the film is littered with hillocks. The inset to Figure 2(a) shows the detail of one hillock that is about $1 \mu \mathrm{m}$ across. Figure 2(b) shows a film grown at $110^{\circ} \mathrm{C}$. The inset in the top left shows that whisker growth is far less profuse than at $125^{\circ} \mathrm{C}$ 
but several well developed whiskers are present. One area is shown enlarged in the main panel and again, there are many hillocks. The top right inset shows an enlargement of another whisker growing from a hillock. This whisker is straight, has clearly facetted sides and a regular cross-section, indicating that is most likely a crystal of a single orientation. Figure 2(c) shows a film deposited at $160^{\circ} \mathrm{C}$. This has a different grain structure and no whiskers. The elevated deposition temperature appears to have enabled sufficient diffusion for the grains to have ripened into well-developed, low surface area crystals and has suppressed the whisker growth. The pattern found on all substrates tested was that by $160^{\circ} \mathrm{C}$, all whisker growth was suppressed.

Given that depositing bismuth by R.F. magnetron sputtering at elevated temperatures leads to the growth of whiskers, the immediate question to be asked is whether this effect would be found in any film regardless of deposition method. We have found that whiskers grow in a similar manner on films deposited by D.C. magnetron sputtering but that none could be seen on films grown by thermal evaporation. We have also investigated deposition of thin films at room temperature using R.F. sputtering followed by subsequent heating to the optimum growth temperature for a similar length of time. In this case, we did not observe whisker growth or change in the diffraction peaks discussed below.

\subsection{X-ray Diffraction}

Several reasons for the difference between sputtering and evaporation may be suggested including differences in film texture or strain. We have investigated the former using X-ray diffraction, Figure 3 shows a comparison between three $\theta / 2 \theta$ X-ray scans; one of a film deposited using R.F. sputtering, one of a film deposited using evaporation and a powder diffraction scan of the evaporation source material for comparison. Looking first at the powder scan, Figure 3 (c), several diffraction orders can be seen clearly; the key ones have been labelled in the rhombohedral scheme [16] on the diagram. The strong diffraction order at $27.19^{\circ}$ corresponds to reflections from the [110] planes. The [111] planes give an order at $22.49^{\circ}$ and the [100] gives a weak peak at $23.79^{\circ}$ and a much stronger [200] at $48.74^{\circ}$. Moving to the two upper panels, (a) and (b), it can be seen immediately that the two deposition processes produce films with different structures. The film produced by evaporation is predominantly [111] textured as can be seen by comparing the relative strengths of the [111] peak and [110] peak to the powder diffraction scan. The film produced by sputtering, however, has an increased [110] content. Note that the [111] and [110] planes in the rhombohedral scheme are [003] and [012] in the hexagonal scheme. Of course, the 
texture can be sensitive to the precise deposition conditions. In this case, conditions were similar: the film produced by evaporation was deposited at a rate of $0.1 \mathrm{nms}^{-1}$ onto a glass substrate held at $115^{\circ} \mathrm{C}$ during deposition and that by sputtering at a rate of $0.12 \mathrm{nms}^{-1}$ onto a glass substrate held at $110^{\circ} \mathrm{C}$.

It is tempting to associate the whisker growth seen in sputtered films with the [110] texture and to contrast this with the predominantly [111] growth in evaporated films where whiskers are not seen. However, the situation is a little more complicated than this. Given that whisker growth is highly sensitive to substrate deposition temperature; it was decided to investigate how this affected the film texture. Figure 4(a) shows the X-ray diffraction scans from sputtered films grown at substrate temperatures between $30^{\circ} \mathrm{C}$ and $160^{\circ} \mathrm{C}$. It can be seen that the degree of [110] texture in the films depends strongly upon growth temperature. Below $125^{\circ} \mathrm{C}$ the predominant texture is [111] though [110] is also present; at $125^{\circ} \mathrm{C}$ and above, the [110] texture dominates. The most profuse whisker growth on glass is seen at $125^{\circ} \mathrm{C}$, where the [110] texture is the strongest. However, at higher temperatures, around $160^{\circ} \mathrm{C}$, no whisker growth is seen despite the continuance of the [110] texture. Note that this discussion refers to the orientation of crystallites in the film and not the orientation of the whiskers.

The difference between sputtered and evaporated films is again shown in Figure 4. Panel (a) shows the X-ray data from the sputtered films and panel (b) the data range of interest from the evaporated films. The evaporated films repeat the main trend of the degree of [110] texture increasing with temperature, however it differs in two key points. The first difference is that at all temperatures, sputtered films show enhanced [110] texture compared with evaporated films. The second difference is that change to [110] dominance occurs at higher temperatures in the evaporated films compared with sputtered films. Thus the temperature dependence of the growth remains something of a puzzle. The optimum temperature for growth may be determined by a complex interplay between the temperature dependence of variables such as thermal strain, diffusion, ripening of nuclei and film crystallite orientation.

\subsection{Influence of Substrates}

So far, the investigations presented here have involved the deposition of bismuth onto glass but several substrates have been tested and all have shown whisker growth within a similar temperature window, though the optimum temperature may change by a few degrees. Two examples of different substrates are illustrated in Figures 5 and 6. Figure 5 shows scanning electron micrographs of $500 \mathrm{~nm}$ bismuth thin films deposited using R.F. sputtering at 0.04 
$\mathrm{nms}^{-1}$ onto a $\mathrm{Si}(100)$ substrate that was held at (a) $30^{\circ} \mathrm{C}$, (b) $110^{\circ} \mathrm{C}$ and (c) $160^{\circ} \mathrm{C}$. The same general trend is seen as for glass, but with some minor differences. The film deposited at $30^{\circ} \mathrm{C}$ shows some poor quality whiskers and fewer large hillocks than found on glass. The inset to Figure 5(a) shows two short whiskers with rough surfaces. Figure 5(b) shows micrographs of a film grown at $110^{\circ} \mathrm{C}$ on $\mathrm{Si}(100)$, close to the optimum temperature for this substrate. The whiskers at this temperature are very straight and well-developed. The inset to the top left is a lower magnification image that shows that some whiskers grow to $30 \mu \mathrm{m}$. Figure 5(c) shows the film grown at $160^{\circ} \mathrm{C}$ illustrating the dramatic change in grain structure and no whiskers. The inset to the top left illustrates a single grain that has two-fold symmetry.

The two whiskers in the centre of the micrograph in Figure 5(b) illustrate an interesting and surprisingly common feature of the films; a whisker cross-over. This feature is enlarged in the inset to the top right of the image. It is a usual feature of these cross-overs that one whisker appears to be indented where the other passes, indicating that they did not grow at the same time. These structures are reminiscent of natural cross-whisker junctions of high temperature superconductors [17]. Given the reports of the observation of superconductivity in low dimensional bismuth [18], such cross-overs may offer a route to fabricate Josephson junctions.

Figure 6 shows the equivalent micrographs as Figure 5 but using a $\mathrm{Si}(111)$ substrate. Despite the change in substrate surface, little difference is seen in the behaviour of the whiskers. Figure 6(a) shows evidence of poor whisker formation at $30^{\circ} \mathrm{C}$. By $110^{\circ} \mathrm{C}$ there are plentiful long straight whiskers (Figure 6(b)), and in the centre is a whisker that has apparently changed growth direction. The inset to the right of Figure 6(b) shows another cross-over. Figure 6(c) shows that whisker formation has again been suppressed by $160^{\circ} \mathrm{C}$ and that the grain structure is changed. The grains in the $160^{\circ} \mathrm{C}$ film on $\mathrm{Si}(111)$ would appear to show a greater number of trigonal crystals than is seen on $\mathrm{Si}(100)$ (Figure 5(c)) but this surface is quite patchy and other regions of the film more closely resemble that on $\mathrm{Si}(100)$

\subsection{Properties of the Whiskers}

Observing the micrographs, the impression is gained that the whiskers have mostly grown at a specific angle to the surface normal; a possibility given the apparent importance of film texture on growth. To investigate the growth angle further, a sample exhibiting good whisker growth was imaged in the electron microscope of a focussed ion beam instrument. This particular substrate was a GaAs(100) wafer. Figure 7(a) to (c) shows three images of the same 
area of this sample with the substrate angle changed in each case successively by $5^{\circ}$. The change in perspective and some fore-shortening effects can be seen clearly in the images. The three images were then combined using the MeX software package [14] into a threedimensional image, which is shown in Figure 7 (d). This image shows clearly that many of the whiskers are at similar angles to the surface normal (note that the software has not been able to recreate the space under the whiskers). The software also enables virtual line scans of the surface; Figure 7(e) shows one such scan along a whisker. The angle that this forms with the surface normal is $47.8^{\circ}$, which is typical. Measurement of several whiskers gave a mean angle to the normal of $54 \pm 3^{\circ}$ with a range between $43.3^{\circ}$ and $69.2^{\circ}$. For rhombohedral bismuth, the angles between the normals to the major planes are as follows: (100)/(110)

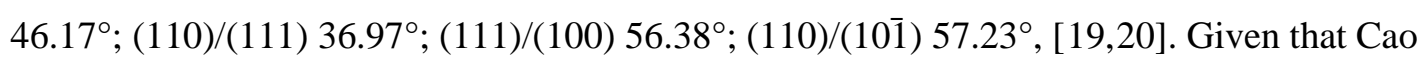
et al reported a whisker growth direction of hexagonal [110] which is equivalent to

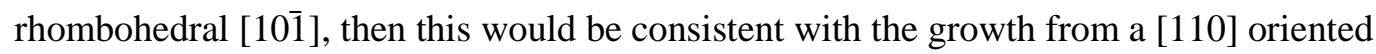
grain as suggested by the $\mathrm{X}$-ray diffraction data. However, given the range of angles this is not conclusive.

Given the prolific nature of their growth at certain temperatures, it is interesting to investigate the length distribution of the whiskers. Figure 8 shows the results of such an investigation on a film of $500 \mathrm{~nm}$ thickness deposited on to a $\mathrm{GaAs}(100)$ substrate held at $110^{\circ} \mathrm{C}$ using $\mathrm{RF}$ sputter deposition at $0.04 \mathrm{nms}^{-1}$. The inset to the graph in the top right corner of the figure is a scanning electron micrograph of the film surface, showing profuse whisker growth. This image was analysed to measure the individual projected length of the individual whiskers. Note that as the micrograph was collected at normal incidence, the measured length is the projection onto the x-y plane; the film surface. If it is the case, as is suspected, that the distribution of the whisker growth angles is around a preferred non-normal direction then this geometrical correction will be constant. If however, the distribution is random then the measurement will be a function of the whisker length and angular distribution.

The bar graph in Figure 8 shows the measured in-plane length of whisker versus the frequency of that measurement in the image. Note that the frequency falls away as the length increases, with the most common projected length being around $8 \mu \mathrm{m}$ but some whiskers exist with lengths greater than $100 \mu \mathrm{m}$. The apparent reduction in frequency for very short whiskers may be an artefact of the difficulty of identifying and measuring vanishingly small lengths. The mean projected length of the measured whiskers is $20 \mu \mathrm{m}$. However, fitting an 
exponential decay to the lengths neglecting those below $6 \mu$ m produces the solid line shown superimposed in Figure 8. The mean projected length of the whiskers from this fitting procedure is $16 \pm 1 \mu \mathrm{m}$, the difference in these two figures may be related to the difficulty of measuring the shortest whiskers. In addition, we have tried to fit a log-normal function to the data in a manner similar to Panashchenko and Osterman [21] but the shape of the curve was less appropriate.

To investigate the transport properties of the whiskers, a film grown on silicon was analysed in an Omicron Nanoprobe [22] based in the Leeds EPSRC National Nanoscience Facility at Leeds University. This device enables four-point measurements to be made on the nano- to micro-scale. Figure 9 shows the measurement of two different whiskers by this technique. Figure 9(a) shows an electron microscope image of a four-point measurement on a whisker in-situ and Figure 9(b) shows the resistance measurement for several different separations of the two (inner) voltage probes. Figure 9(c) and (d) shows the equivalent data for a different whisker. Estimating the probe separations and cross-section of the whisker from the scanning electron microscope images, the gradient of Figure 9(b) gave a whisker resistivity of $2.3 \pm 0.2$ $\mu \Omega \mathrm{m}$, a similar analysis on Figure 9(d) gave a resistivity of $2.2 \pm 0.2 \mu \Omega \mathrm{m}$. Both measurements lie between the bulk resistivity of bismuth, which is $1.3 \mu \Omega \mathrm{m}[23,24]$ and the recently reported resistivity of a $127 \mathrm{~nm}$ diameter nanowire which was $3.9 \mu \Omega \mathrm{m}$ [25]. When comparing these values there are two notes of caution; the first is that the resistivity of bismuth is anisotropic [24] which would be an important issue in single crystal whiskers, the second is that for the nanowires and whiskers the surface would be prone to oxidation, increasing the apparent resistivity.

\subsection{General Discussion}

In considering the possible mechanisms of growth of these whiskers, it is important to distinguish them from the whiskers that are common on other low melting point metals in, for example, electronic components. Usually whiskers root-grow over prolonged periods of time from thick films, driven by residual stress. Whilst it is true that this growth method can be accelerated by the use of elevated temperatures it is clearly different from the mechanism that is at work in the sputter-deposited films. We believe that the whiskers develop during the thin film growth, which takes times up to one hour, and we have not been able to grow whiskers from thin films by using post-deposition heating, even for many hours. Thus the mechanism 
that creates the whiskers during deposition is distinct from the more usual mechanism and is related to the growth.

There are several reported methods for engineering the growth of whiskers and nanowires on bismuth films. There was an early report [26] of the growth of whiskers on bismuth thin films using evaporation deposition. The procedure for this was quite different from the sputter deposition method reported here. A $50 \mathrm{~nm}$ bismuth film was evaporated onto a pre-existing manganese $(200 \mathrm{~nm})$ film. This was followed by a post-deposition heating for three to four hours at temperatures around $250^{\circ} \mathrm{C}$. Whilst the temperatures and time-scales are comparable with those used in the sputter-deposition method, the authors of this report stated that whiskers grew only in the presence of the manganese sub-layer. However, in the work reported here we have not used another element to induce the whisker growth and we have not been able to produce whiskers by evaporation deposition with or without post-deposition heating.

A more recent report regarding the growth of bismuth nanowires also involved co-deposition [27]. This method involves the sputter-deposition at room temperature of a nano-composite thin film of chromium-nitride containing a few atomic percent of bismuth. The intrinsic stress in the freshly deposited film drives the growth of bismuth nanowires by root growth. The electron micrographs of these nanowires show that they are different in character from the whiskers that appear spontaneously on sputter deposited films.

Another stress-driven thin-film mechanism, the off-on method, has been reported [28,29]. This involves post-deposition heating to $260^{\circ}-270^{\circ}$ for ten hours of a bismuth thin film sputter-deposited at room temperature onto an oxide film on a $\mathrm{Si}(100)$ substrate. The heating induces stress in the film which is relaxed by extrusion of nanowires normally to the film. The time-scale of this is much longer than that used here and the substrates were pre-etched to remove the oxide.

There are several methods known to tip-grow grow whiskers, nanorods and nanowires of materials other than bismuth. The vapour-liquid-solid method (VLS) [30,31], is frequently used to grow elemental and compound semiconductor nanowires. In this method, the substrate is pre-dosed with gold or a similar metal that is then assembled into nano-dots by substrate heating. Under a growth flux of the semiconductor, these dots then catalyse the growth and "float up" on the ends of the nanowires. Whilst it is possible that there are some 
similarities in the growth mechanism between this and that of our bismuth nanorods we have not used a catalyst and the wires grow from the elemental bismuth itself.

A known variant of the VLS method involves self-catalysation [32], which is most commonly found where nanowires of a compound semiconductor are grown reactively. Typically, the self-catalyst is a liquid bead of a low melting-point metal that forms a higher melting point solid compound on reaction with the incoming flux. This requires the substrate to be held at a temperature between the two melting points. There are also methods of nanowire growth that may be loosely classified as vapour solid (VS) growth. These often resemble chemical vapour deposition in that an element may be deposited from a compound gas, for example the growth of copper nanowires [33] from $\mathrm{CuCl}$ gas. VS is therefore often a reactive process.

There is of course a natural tendency for some materials to grow prismatic crystals. An example is the reactive growth of hexagonal prismatic $\mathrm{ZnO}$ whiskers in a furnace environment [34]. The authors of this work suggested that the mechanism of growth was related to the tendency of some faces in the hexagonal system to grow more quickly than others [35]. Bismuth is of course hexagonal and would therefore also be expected to exhibit uniaxial asymmetries in growth rate.

As stated previously, the spontaneous growth of whiskers on our films is similar to that reported by Cao et al [13], though at an apparently lower temperature. They excluded stress as a driver and proposed instead a tip-growth vapour-solid mechanism induced by screw dislocations in film grains. In this model, the whiskers grow helically around the dislocation during deposition.

In considering possible models for the growth of the bismuth whiskers there are several pieces of evidence to be considered. One of these is the tendency of the whiskers to grow at a non-normal angle from the surface. This would appear to rule out a mechanism similar to the off-on method [28] for producing nanowires by a stress driven mechanism. Indeed the apparently quite specific angles of growth relative to the film crystallites hint at a growth mechanism rather than an extrusion mechanism. If extrusion is to be excluded then these whiskers differ in nature to those commonly found on low melting point metals.

The whiskers are highly uniform in width and remarkably straight, which would also indicate a crystal growth mechanism. That the growth is particularly dependent on temperature also 
points to diffusion having an important role to play. That we only observe them in sputterdeposited films rather evaporated films would at first seem to indicate a stress related mechanism but root growth can be ruled out by the cross-overs, examples of which are shown in figure 5(b) and figure 6(b) insets right. Thus the sputter-deposition environment must also influence the tip growth, either by seeding the growth with appropriate crystals, by changing the energy of the deposited atoms, or by the influence of plasma leaking from the magnetron to the substrate.

Careful examination of the micrographs also reveals some important features of the whiskers: they often have striations around them, see for example figure 5(b); they usually have faceted ends figure 10(a-c) but are occasionally more rounded; they sometimes exhibit bends through what appears to be a specific angle, for example the one shown in figure 10(d), consistent with a crystal growth phenomenon; they frequently exhibit cross-overs where one whisker is indented at the junction but then recovers its diameter figures5(b) and 6(b) insets right; a very few contain grooves that run the full length of the whisker, for example figure 6(b) inset right.

Given that the mechanism must involve tip growth, this requires faster growth on one crystal plane than on the others; in fact this is what methods such as VLS, the screw dislocation model and others achieve. The sensitivity to growth temperature suggests that the competition between diffusion and deposition rate is important. The screw dislocation model proposed by Cao et al [13] is sensible and is supported by their micrographs. However, it is difficult to see why this model applies only to sputter deposited films and not evaporated films. It cannot be the mechanism by which some of our whiskers grow, as can be seen in figure 6(b). The pair of grooves in the side of the whisker appears to rule out spiral growth modes for this particular whisker, though others such as in Figure 10(f) resemble the ones shown in reference [13].

A VLS type mechanism would be a possibility except for the lack of a catalyst. Selfcatalysation should not work at these deposition temperatures as the substrate is below the melting temperature for bismuth. However, the surface temperature may actually be a little higher due to the energy supplied by the deposition process, especially at the tips of asperities. Most whiskers and nanowires grown by VLS have rounded tips. The micrographs in figure 10 show that most, but not all, of the tips of our whiskers are faceted. Of course it may be that slow cooling of molten bismuth on a bismuth single crystal results in crystallisation of the 
melt and faceting driven by energy minimisation. However, despite the faceting the general shape of the tips is hemispherical.

The low deposition rate found when sputtering bismuth onto a hot substrate implies that the process has a competition between condensation and evaporation. This could accentuate small differences in growth rate between surfaces. However, a similar competition may be expected when the films are deposited by evaporation.

The cross-overs illustrated in figures 5(b) and 6(b) are one of the most interesting features of the growth. The apparent indenting on one whisker around the other suggests that the two whiskers do not grow simultaneously. The growth mechanism allows the later one to recover its girth after the cross, consistent with a VLS method if the molten bead is retained. However, the cross-overs also lend support to a relative growth rate model. We have shown (later) that the whiskers on thinner films are themselves narrower, suggesting that the whiskers grow thicker with time as well as longer. This would support a generalised model based on differing growth rates for each face.

So, in considering which model to propose for the formation of the whiskers, it would appear that a tip-growth rather than root-extrusion is appropriate. This tip growth must result from an accelerated growth in one crystal direction due to a non-catalysed VLS/VS process. The details of the process are probably quite subtle and require the higher energy of a sputterdeposition process as opposed to evaporation. Getting the growth conditions just right must require a balancing of the competitions between deposition, diffusion and re-evaporation. Processes at the tip, possibly including energy delivered by plasma leaking from the magnetron, accelerate the growth in that direction. That many of these whiskers grow around a screw dislocation would be sensible as such defects are known to accelerate growth, but it appears that not all of them do.

\subsection{Production of Nanowires}

Whilst the growth of whiskers is interesting, both as a way of obtaining high quality, high aspect ratio bismuth crystals and for the implications for the increasing use of bismuth in electrical solders, one of the growth areas in research into bismuth is the production of nanowires: that is long, uniform structures with diameters less than $100 \mathrm{~nm}$. To investigate the utility of sputtering at temperature in the production of nanowires we deposited some thinner films, specifically $100 \mathrm{~nm}$ thick. Figure 11 shows scanning electron micrographs of 
such thinner films, this time deposited using low power DC magnetron sputtering. It can be seen that the whiskers that grow in this case are both sparser and narrower, a typical diameter being around $100 \mathrm{~nm}$. Given that a $500 \mathrm{~nm}$ film grows $500 \mathrm{~nm}$ diameter whiskers and a 100 $\mathrm{nm}$ film grows $100 \mathrm{~nm}$ ones, it is intriguing to surmise that the diameter of the whisker may be controllable in this way to produce nanorods and nanowires of a particular desired size.

Figure 11 shows examples of nanowires on a $100 \mathrm{~nm}$ bismuth film grown using $30 \mathrm{~W}$ of DC sputtering onto a $\mathrm{Si}(111)$ wafer. The example shown in figure 11(a) \& (b) has length of 12.7 $\mu \mathrm{m}$ and diameter of $80.0 \mathrm{~nm}$. Other examples of nanowires are shown in figure 10(c); the central one has length of the order $20 \mu \mathrm{m}$. Note that these nanowires have such a large aspect ratio that they are unable to maintain the straightness that is found in wider whiskers. An initial investigation of these nanowires suggests that slow growth promotes increased length.

\section{Conclusion}

We have shown that bismuth whiskers grow spontaneously on a thin film produced by planar magnetron sputtering when the substrate is heated to a suitable temperature during deposition. Optimum temperatures for such growth are between $110^{\circ} \mathrm{C}$ and $140^{\circ} \mathrm{C}$ and are largely independent of substrate. Depositing films under similar conditions but by thermal evaporation does not produce the whisker growth. X-ray diffraction has been employed to investigate film texture with temperature. It has been shown that the crystallites are predominantly [110] and [111] oriented; with the latter dominating at lower deposition temperatures and the former at higher temperatures. The optimum temperature for growth coincides with the temperature for the switch between predominant orientations. Sputter deposition enhances the [110] texture over the [111] when compared with evaporation deposition.

Analysis of the whiskers has shown that they mostly emerge at off-normal angles between $43.3^{\circ}$ and $69.2^{\circ}$ with a mean of $54 \pm 3^{\circ}$.The projected length of whiskers on a $500 \mathrm{~nm}$ film shows a wide distribution from a mode at around $1 \mu \mathrm{m}$ to a maximum of more than $100 \mu \mathrm{m}$. The mean projected length for this sample was $16 \pm 1 \mu \mathrm{m}$ and the diameter is around $0.5 \mu \mathrm{m}$. Measurements of the electrical properties of the whiskers at room temperature reveals ohmic behaviour with an estimated resistivity of $2.2 \pm 0.2 \mu \Omega \mathrm{m}$.

Detailed examination of the micrographs eliminates all mechanisms for growth except tip growth by a non-catalysed VLS/VS method. Finally, by depositing thinner films we have shown that this spontaneous growth of whiskers offers a route to fabricate bismuth nanowires 
of lengths exceeding $10 \mu \mathrm{m}$. These nanowires appear to be of excellent uniformity and would in all probability be single crystal.

\section{Acknowledgements}

Several people and organisations have made this research possible. The electron microscopy was performed by John Bates, Geoff West and Keith Yendall of the Department of Materials at Loughborough University. The four point probe measurements were performed at the Leeds EPSRC Nanoscience and Nanotechnology Facility and the EPSRC is thanked for providing access. Simon Halse helped with the literature search and Technical support at Loughborough was provided, as ever, by Bryan Dennis. The two anonymous referees are thanked for their constructive suggestions. 


\section{References}

[1] See for example Galyon G T, Annotated Tin Whisker Bibliography, National Electronics Manufacturing Initiative Publication, pp. 1-22, Updated July 2003 (orig. Feb. 2003)

[2] Peach M O, (1952) Journal of Applied Physics 231401

[3] Frank F C, (1953) Philosophical Magazine XLIV 854

[4] Eshelby J D, (1953) Physical Review 91755

[5] Koonce S E and Arnold S M, (1953) Journal of Applied Physics 24365

[6] Lee B Z and Lee D N (1998) Acta Materialia 463701

[7] Lin Y M, Sun X, Dresselhaus M S, (2000) Physical Review B 624610

[8] Hicks L D, Dresselhaus M S, (1993) Physical Review B 4712727

[9] Gitsu D, Konopo L, Niklaeva A, Huber T E, (2005) Applied Physics Letters 86102105

[10] Chiu P, Shih I, Nanotechnology (2004) 151489

[11] Heremans J, Thrush C M, Lin Y M, Cronin S, Zhang, Dresselhaus M S, Mansfield J F, Physical Review B (2000) 612921

[12] Cronin S B, Lin Y M, Rabin O, Black M R, Dresselhaus G, Dresselhaus M S, Gai P L, (2002) Microscopy and Microanalysis 858

[13] Cao S, Guo C, Wang Y, Miao J, Zhang Z, Liu Q, (2009) Solid State Communications 14987

[14] MeX package produced by Alicona, Austria [http://www.alicona.com]

[15] Kim D-H, Lee S-H, Kim J-K, Lee G-H, (2006) Applied Surface Science 2523525

[16] Wykoff R W G, Crystal Structures Vol. 1, Second Edition, New York, Interscience, 1963.

[17] Latyshev Y I, Orlov A P, Nikitina A M, Monceau P and Klemm R A, 2004 Physical Review B 70094517

[18] Tian M, Wang J, Kumar N, Han, T, Kobayashi Y, Liu Y, Mallouk T E, Chan, M H W, (2006) Nano Letters 62773

[19] Salkovitz E I, 1956 Transactions of the American Institute of Mining and Metallurgical Engineers 206176

[20] Bacon D J, Hecksher F, Crocker A G, (1964) Acta Crystallographica 17760

[21] Panashchenko L, Osterman M, (2009) Electronic Components and Technologies Conference (ECTC), 1037

[22] Walton A S, Allen C S, Critchley K, G’orzny M Ł, McKendry J E, Brydson R M D, Hickey B J and Evans S D, (2007) Nanotechnology 18065204

[23] Source: WebElements [http://www.webelements.com/]

[24] Gallo C F, Chandrasekhar B S, Sutter P H, (1963) Journal of Applied Physics 34144

[25] Lee S, Ham J, Jeon K, Noh J-S, Lee W, (2010) Nanotechnology 21405701

[26] Mayer L, Rickett R, Stenemann H, (1962) Journal of Applied Physics 33982

[27] Cheng YT, Weiner A M, Wong C A, Balogh M P, Lukitsch M J, (2002) Applied Physics Letters 813248.

[28] Shim W, Ham J, Lee K, Jeung W Y, Johnson M, Lee W, (2009) Nanoletters 918.

[29] Shim W, Ham J, Kim J, and Lee W, (2009) Applied Physics Letters 95, 232107.

[30] Wagner R S, Ellis W C, (1964) Applied Physics Letters 489

[31] Hiruma K, Yazawa M, Katsuyama T, Ogawa K, Haraguchi K, Koguchi M, Kakibayashi H, (1995) Journal of Applied Physics 77447 
[32] Hsu-C-L, Chang S-J, Hung H-C, Lin Y-R, Huang C-J, Tseng Y-K, Chen I-C, (2005) IEEE Transactions on Nanotechnology 4649

[33] Yen M-Y, Chiu C-W, Hsia C-H, Chen F-R, Kai J-J, Lee C-Y, Chiu H-T, (2003) Advanced Materials 15235

[34] Hu J Q, Li Q, Wong N B, Lee C S, Lee S T, (2002) Chemistry of Materials 141216

[35] Laudise R A, Ballman A A, (1960) Journal of Physical Chemistry 64688 


\section{Figure Captions}

Figure 1: (a) and (b) show scanning electron microscope images of a whisker on a bismuth film grown by RF sputtering onto glass. The film is $400 \mathrm{~nm}$ thick and was deposited at a rate of $0.04 \mathrm{nms}^{-1}$ with a substrate temperature of $145^{\circ} \mathrm{C}$. Figure 1 (c) shows a lower magnification image of a $500 \mathrm{~nm}$ film grown at $0.12 \mathrm{nms}^{-1}$ on a glass substrate held at $125^{\circ} \mathrm{C}$ during deposition.

Figure 2: Scanning electron microscope images of bismuth films grown by RF sputtering at $0.04 \mathrm{nms}^{-1}$ onto glass substrates held at different temperatures: (a) $30^{\circ} \mathrm{C}$; (b) $110^{\circ} \mathrm{C}$, (c) $160^{\circ} \mathrm{C}$. The insets highlight features in the films.

Figure 3: Comparison of theta/two-theta X-ray diffraction scans from bismuth films deposited by (a) evaporation at $0.1 \mathrm{nms}^{-1}$ onto glass substrates held at $115^{\circ} \mathrm{C}$ during deposition and (b) RF sputtering at $0.12 \mathrm{nms}^{-1}$ onto glass substrates held at $110^{\circ} \mathrm{C}$. The powder diffraction scan collected from the evaporation source material is shown for comparison in (c).

Figure 4: Theta/two-theta X-ray diffraction scans from bismuth films deposited by (a) RF sputtering at $0.12 \mathrm{nms}^{-1}$ onto glass substrates held at different temperatures during deposition. (b) The data for the $20^{\circ}-30^{\circ}$ angular range for evaporated films are shown for comparison. Two curves had been made grey to avoid obscuring other curves.

Figure 5: Scanning electron micrographs of $500 \mathrm{~nm}$ bismuth thin films deposited using RF sputtering at $0.04 \mathrm{nms}^{-1}$ with the $\mathrm{Si}(100)$ substrate held at (a) $30^{\circ} \mathrm{C}$, (b) $110^{\circ} \mathrm{C}$ and (c) $160^{\circ} \mathrm{C}$. The insets highlight features in each film.

Figure 6: Scanning electron micrographs of $500 \mathrm{~nm}$ bismuth thin films deposited using RF sputtering at $0.04 \mathrm{nms}^{-1}$ with the $\mathrm{Si}(111)$ substrate held at (a) $30^{\circ} \mathrm{C}$, (b) $110^{\circ} \mathrm{C}$ and (c) $160^{\circ} \mathrm{C}$. The insets highlight features in each film.

Figure 7: The angle of a typical whisker to the surface normal. Three electron micrographs collected from a bismuth film at three angles differing by plus and minus $5^{\circ}$ (a), (b), and (c). 
These have been rendered into a three-dimensional image (d) and a profile scan of a whisker (e).

Figure 8: The length distribution of bismuth whiskers imaged on a film of $500 \mathrm{~nm}$ thickness deposited on to a GaAs(100) substrate held at $110^{\circ} \mathrm{C}$ using RF sputter deposition at 0.04 $\mathrm{nms}^{-1}$. The inset to the graph shows the scanning electron micrograph of the film and the bargraph itself the number distribution for the length of whiskers within the image. The solid line in the graph is the result of a fitting procedure discussed in the text.

Figure 9: Nanoprobe measurements of the resistivity of two whiskers. Panel (a) shows a whisker with four probes attached. Panel (b) shows the variation in resistance as the two central probes are moved. Panels $\quad$ (c) and (d) show the same for a different whisker.

Figure 10: Micrographs showing the details of growth of some of the whiskers.

Figure 11: Scanning electron micrographs of a $100 \mathrm{~nm}$ thick bismuth film grown using $30 \mathrm{~W}$ of DC sputtering onto a Si(111) wafer. Panel (a) shows a nanowire of $12.7 \mu \mathrm{m}$ length. Panel (b) shows the same nanowire enlarged to reveal a diameter of $80.0 \mathrm{~nm}$. Panel (c) shows another long nanowire. 


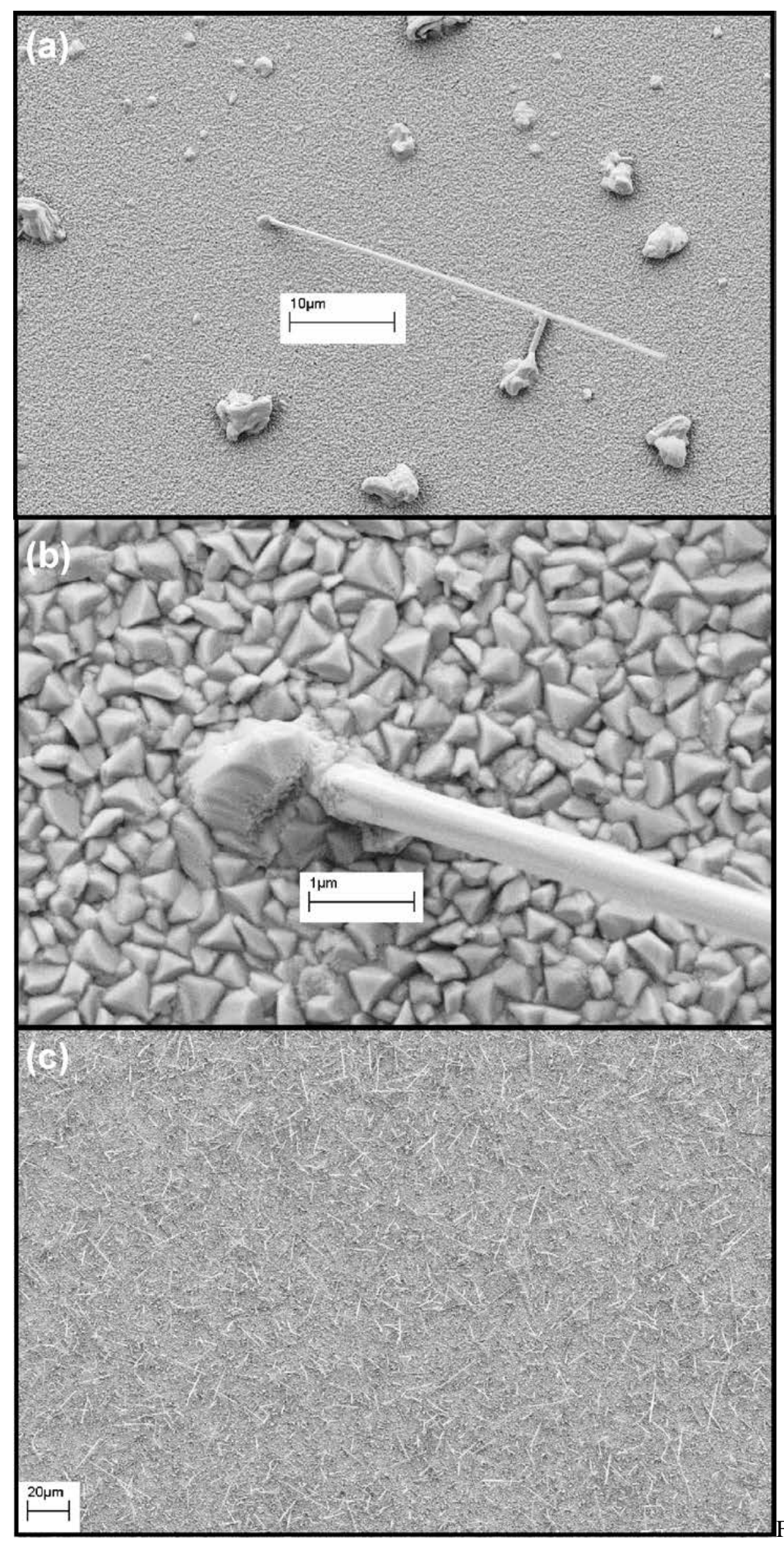



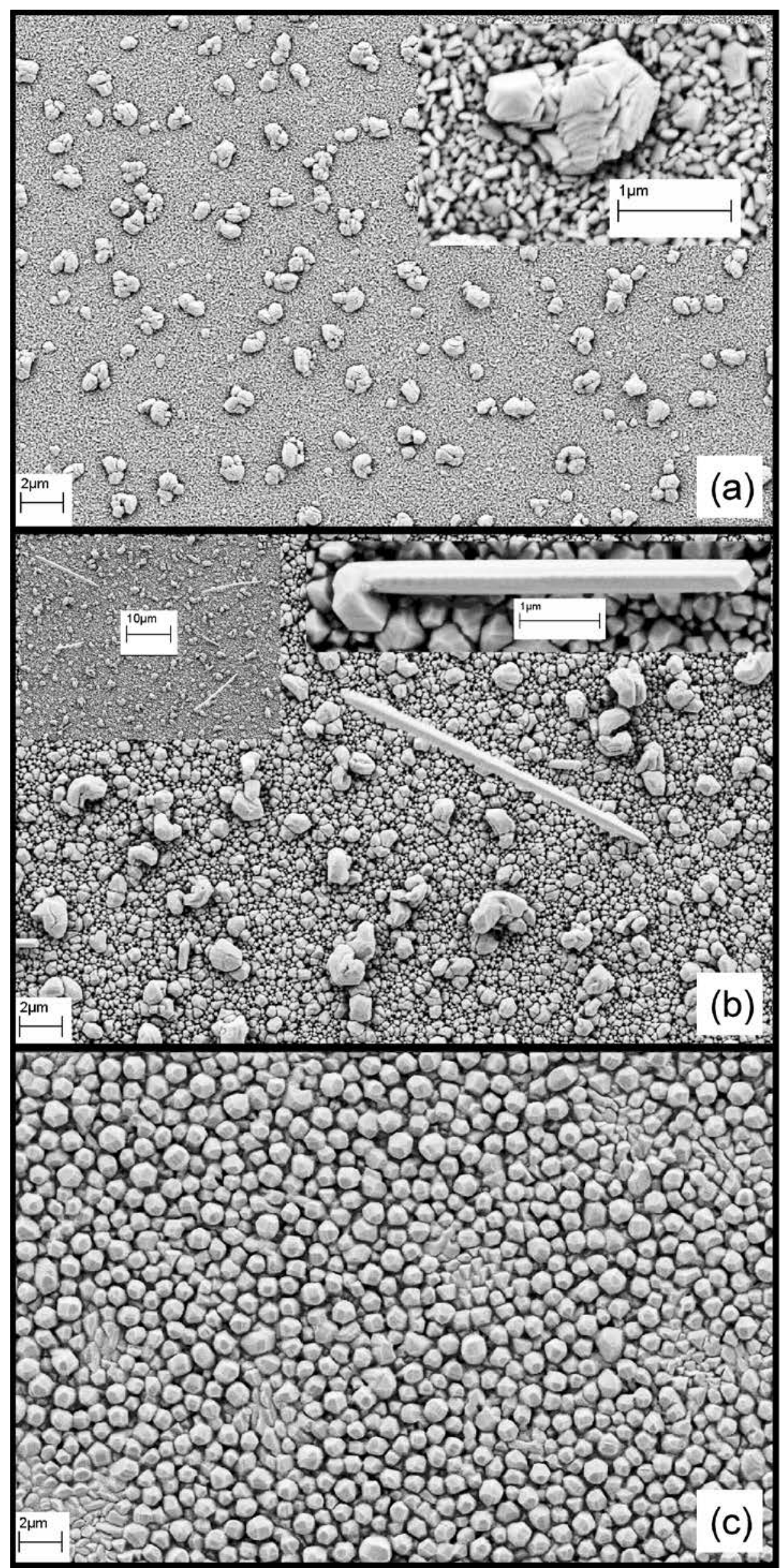


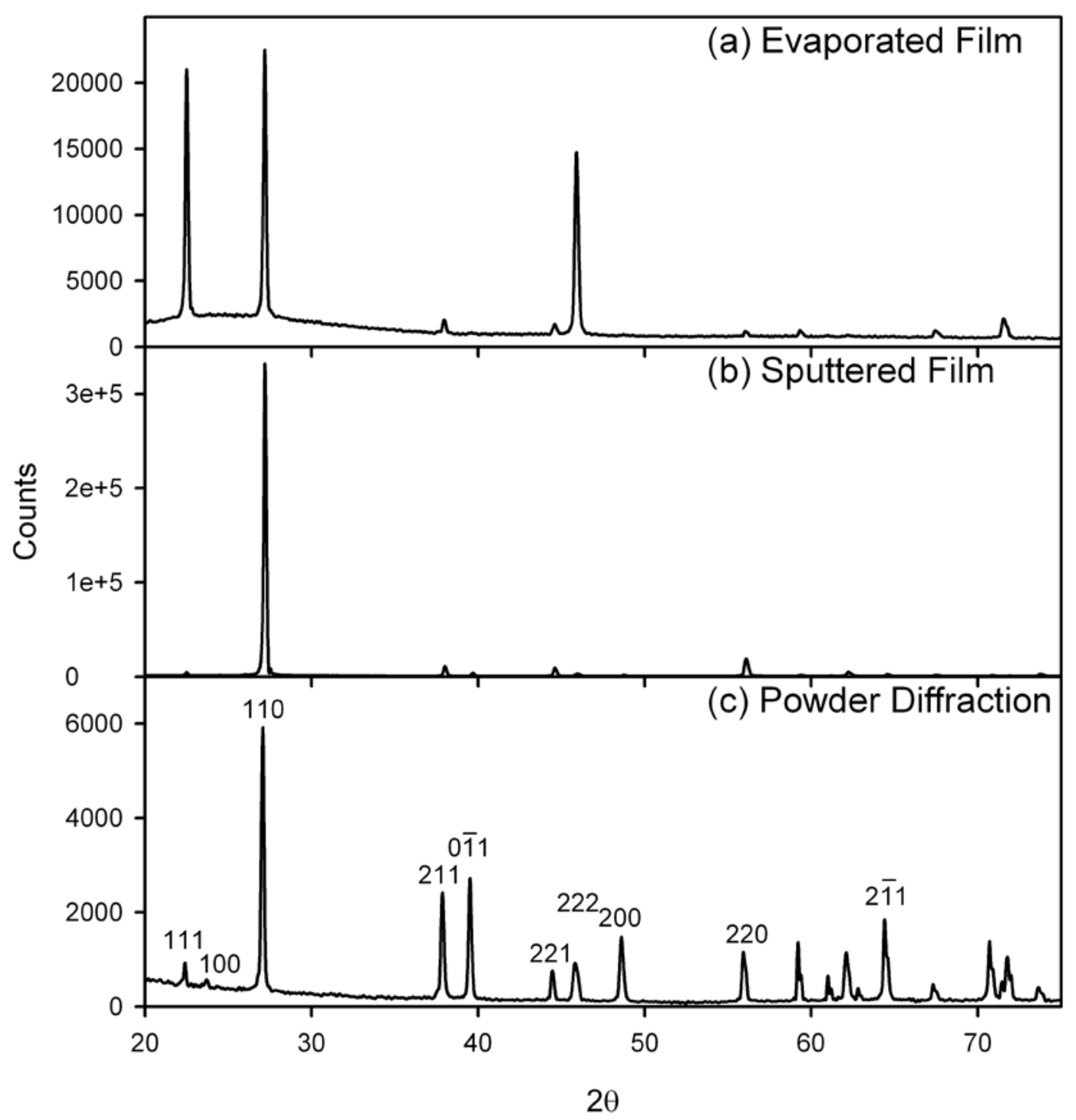

Fig 3 


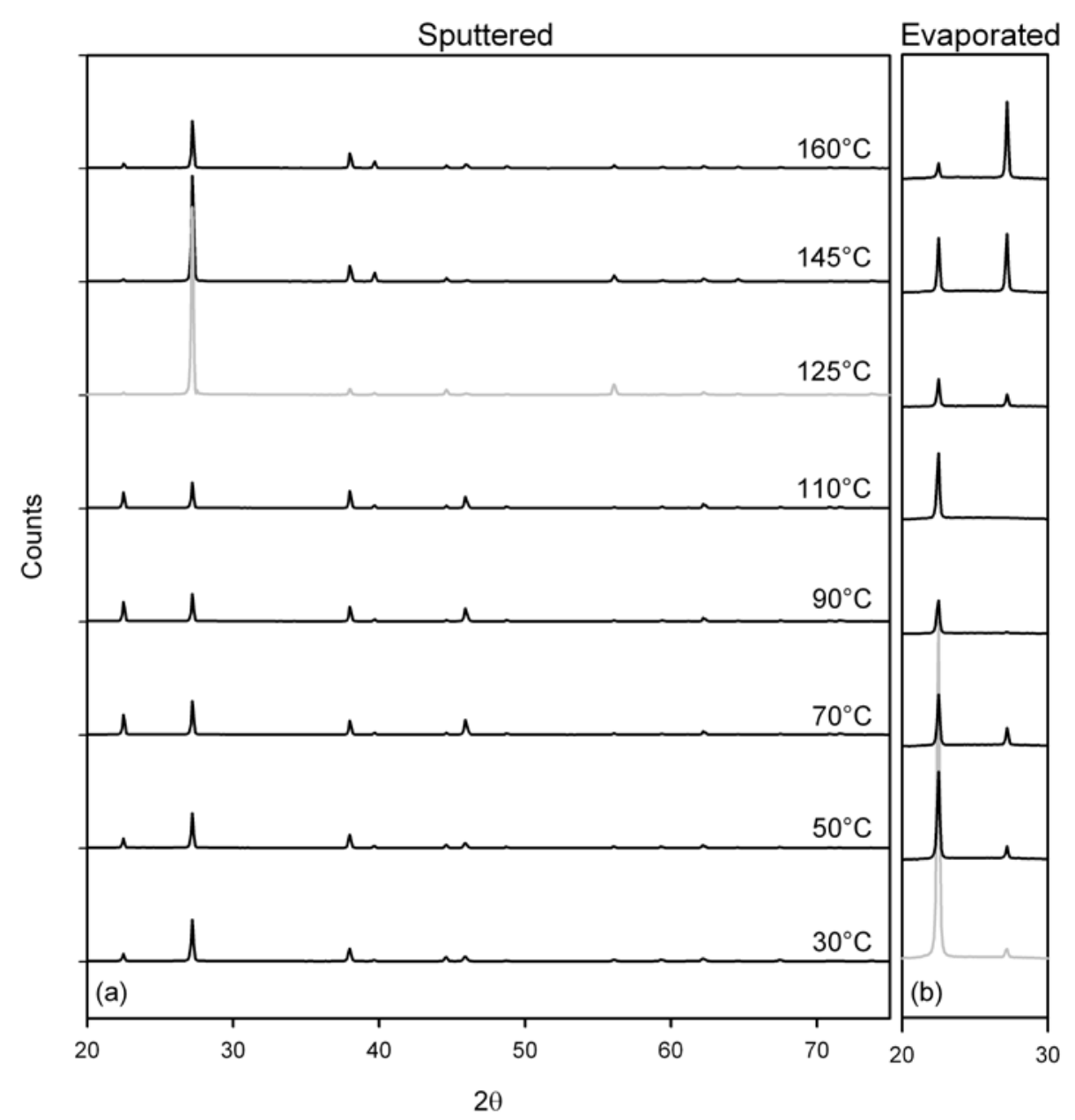

Fig 4 


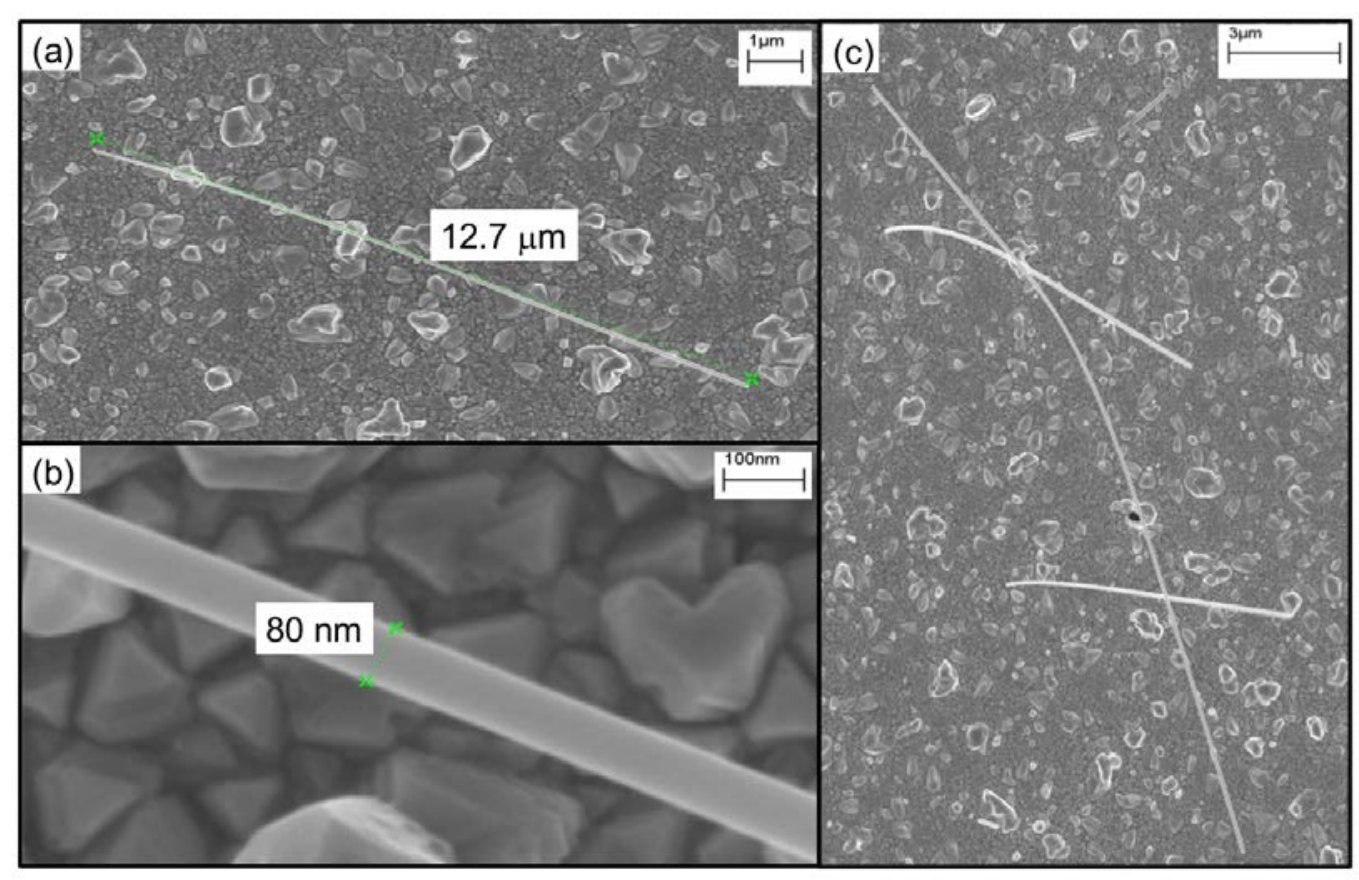

